\title{
Positive Affect, Negative Affect, and Social Interaction
}

\author{
Diane S. Berry and Jane Sherman Hansen \\ Southern Methodist University
}

\begin{abstract}
Two studies explored the relations of positive and negative affect (PA and NA) to social interaction. In Study 1, unacquainted dyads were surreptitiously videotaped as they participated in a 6-min interaction. Participants then evaluated the quality of the interaction. Independent observers also rated the videotaped interactions. Trait PA was positively related to both participant and observer evaluations of interaction quality. In Study 2, undergraduates kept diaries of their social interactions for 1 week. PA was again related to interaction quality. Both PA and NA were positively related to the number of interactions in which participants engaged, and the amount of time spent engaged in social contact, although different types of social encounters produced these relations.
\end{abstract}

Psychologists have long been interested in individual differences in people's self-described emotional experience. Research has revealed that some people report experiencing greater amounts of positive emotions than do others and are typically referred to as high-positive-affect ( or high-PA) individuals. People who are high in positive affect are, for example, consistently more likely to describe themselves as enthusiastic, confident, and excited than are people who are low in positive affect. On the other hand, some individuals' daily lives are characterized by greater amounts of negative affective states than are others, and they are often referred to as high-negative-affect (or high$N A$ ) people. For example, people who are high in NA are more likely to describe themselves as guilty, fearful, and nervous than are low-NA individuals. Although there are fluctuations in the levels of positive or negative affect a given person experiences at different points in time, the stability of self-reports of NA and PA is impressive (e.g., Watson, 1988a, 1988b). In sum, there appear to be traitlike interindividual differences in people's typical self-reported levels of NA and PA.

These individual differences in trait NA and PA also seem to underlie the personality dimensions of neuroticism and extraversion. In particular, self-report measures of NA and neuroticism have been repeatedly demonstrated to be highly correlated with one another but unrelated to measures of either positive affect or extraversion. Similarly, PA and extraversion are strongly related but are uncorrelated with either NA or neurot-

Diane S. Berry and Jane Sherman Hansen, Department of Psychology, Southern Methodist University.

Portions of these data were presented at the Nags Head Conference on Close Relationships, Highland Beach, Florida, June 1994, and at the meeting of the International Network on Personal Relationships, Williamshurg, Virginia, June 1995. We are grateful to Ephrem Fernandez, Jamie Pennebaker, and especially, David Watson for their very thoughtful suggestions on this work. We also thank Gretchen Jones for her assistance with the data collection in Study 1, Julie Landry for collecting the diary data featured in Study 2, and Bill Ickes for generously providing advice regarding the data analyses reported in Study 1 .

Correspondence concerning this article should be addressed to Diane S. Berry, Department of Psychology, Southern Methodist University, Dallas, Texas 75275 . icism. This pattern of relations has been repeatedly observed (e.g., Costa \& McCrae, 1980; Emmons \& Diener, 1985; Watson, Clark, McIntyre, \& Hamaker, 1992), leading some researchers to propose that the personality factors of neuroticism and extraversion stem from stable individual differences in people's susceptibility to the experience of negative and positive affective states, respectively (e.g., Watson et al., 1992; Watson \& Clark, 1992).

One might be inclined to expect substantial covariation between these positive and negative emotion traits. For example, clinical depression is characterized by both intense negative feelings and the marked absence of positive emotions (e.g., Beck, 1967). Similarly, there is a strong negative correlation between observer ratings of the extent to which other people express positive and negative affect (Berry \& Pennebaker, 1993, in press; Berry, Pennebaker, \& Mueller, 1996). However, research has repeatedly established that self-reports of the tendency to experience NA and PA within nonclinical populations are relatively independent, with relations typically in the -.1 to -.3 range (e.g., Diener \& Emmons, 1984; Watson, 1988a, $1988 \mathrm{~b}$ ). Thus, people who report that they often feel enthusiastic, energetic, and excited may or may not often feel quite hostile, anxious, and guilty. In fact, this is one of the features that distinguishes both positive affect and negative affect from dimensions such as depression (Watson \& Clark, 1984). NA and PA have further been found to bear independent relations to a variety of self-report items. For example, NA is related to selfreports of health complaints, whereas PA is not (Watson \& Pennebaker, 1989). On the other hand, PA is related to self-reports of social activity, whereas NA generally is not (e.g., Watson, 1988a; Watson et al., 1992).

Several studies have revealed links between positive affect and social activity. Watson (1988a) asked participants to estimate the number of hours per day that they spent with friends. Measures of their levels of trait NA and PA also were obtained. Analyses revealed that trait PA was positively correlated with, and NA was unrelated to, the mean number of hours of daily social contact that people reported. In a pair of follow-up studies, Watson et al. (1992) assessed the relations between NA and PA and more specific types of social activity. In Study 1, participants filled out a questionnaire on which they estimated how often 
they had participated in each of 15 different kinds of social activities during the previous week (e.g., playing cards, having a conversation). Trait PA was found to be positively related to self-reported frequency of social activity, whereas trait NA was unrelated to these measures. In Study 2, participants completed daily questionnaires on which they indicated the amount of time that they had spent involved in each of 21 social activities. Again, PA was positively related to the amount of various sorts of social activities in which people engaged. Unexpectedly, NA was also positively related to self-reported social contact.

These data are provocative and raise as many questions as they answer. First, previous work has largely focused on estimates of the amount of time spent in, or the frequency of engagement in, social activity. However, people's social experiences come in very different shapes and sizes, and virtually nothing is known about possible relations between affect and other aspects of social interaction. For example, with whom is this time spent? Does the correlation between positive affect and social contact mean that high-PA individuals participate in numerous same-sex group activities, enjoy long hours in the company of one member of the opposite sex, or both? We also know very little about affective style and qualitative aspects of social interaction. If NA and PA are basic dimensions of personality, it seems likely that each influences the nature of people's social experiences. However, most of the available data focus on the relations of these dimensions to quantitative aspects of social activity. In fact, we located only one report that directly examined the links between NA and PA and qualitative assessments of people's interactions. Cooper, Okamura, and Gurka (1992) compared people's levels of NA and PA to their general satisfaction with their social activities in two different samples. The results were mixed. In one sample, neither measure predicted satisfaction. In a second sample, PA was positively related to, and NA was negatively related to, reported satisfaction. In sum, little is known about the relation of positive and negative affect to either the frequency of different kinds of social contact or to qualitative aspects of various types of social interactions.

A second issue that warrants attention is the exploration of the mechanisms that may produce links between affect and interaction. For example, why would PA be related to interaction frequency? Researchers have typically focused on direct explanations of the link between PA and sociability. In particular, this relation has been proposed to result from high-PA individuals' acute sensitivity to potentially pleasurable stimuli, including social stimuli (e.g., Tellegen, 1985). If this is indeed the case, those who are high in PA may derive especially high levels of pleasure from social contact; a given social encounter may actually feel qualitatively better to someone high in trait PA than to someone else. The enjoyment that high-PA individuals derive from social interaction may reinforce their already heightened level of positive feelings and in turn their desire for additional social contact (cf. Watson et al., 1992).

An alternative explanation of the relation between PA and social contact that has received less attention focuses on differences in the social constraints that may accrue to people who have different affective styles. If interactions with high-PA individuals are particularly enjoyable, others may be especially likely to seek out their company, thus increasing the opportunities for social activity afforded to people who are high in trait
PA. This account differs from that previously discussed in that it does not posit any direct link between affect and sociability but instead points to indirect links produced by differences in the social opportunities elicited by one's level of PA. How might we evaluate the potential merit of a social constraint explanation of the relations between affect and sociability? The direct explanation assumes only that high-PA individuals derive greater enjoyment from social interaction than do other people. However, the social constraint account of these links further requires that other people perceive high-PA individuals to be especially rewarding social partners and therefore worth engaging in social activity. Thus, the latter view suggests that high-PA individuals' interactions should be "objectively" better than those of low-PA individuals and that this difference be discernible to other people. To date, no research has addressed this issue.

Finally, we should note that previous work in this area has typically involved the use of global, retrospective descriptions of interaction frequency and quality. Some researchers have critiqued such measures as being susceptible to bias (cf. Reis \& Wheeler, 1991). Moreover, studies in which such measures of quantitative aspects of peoples' interactions have been compared to some objective standard have raised questions about the measures' accuracy (e.g., Conrath, Higgins, \& McClean, 1983). Although this suggests that retrospective estimates of interaction frequency are probably not the most reliable measure of social activity, it should be noted that this is less of a problem in studies in which such accounts are provided frequently (e.g., daily; Watson et al., 1992, Study 2) than in studies in which these reports are sampled less often. Similar concerns may be raised about assessments of qualitative aspects of social interactions. For example, people may recall their social encounters in a more favorable light when they are in a positive emotional state than when they are experiencing negative affect (e.g., Forgas, Bower, \& Krantz, 1984). Again, such problems are especially likely when people provide general assessments of their social lives as opposed to descriptions of particular social experiences. One alternative that eliminates such problems involves obtaining "on-line" assessments of specific social interactions in which people engage. For example, data from studies that have used social interaction diaries indicate that such a strategy yields highly reliable assessments of interaction patterns (e.g., Hodgins \& Zuckerman, 1990). Thus, the essentially on-line behavior recordings used in an approach like this do appear to provide a clearer view of one's social experience than is made available by retrospective global estimates.

We report two studies in which we addressed the issues discussed above. In Study 1 we randomly assigned 112 previously unacquainted women to dyads and surreptitiously videotaped them as they engaged in an unstructured initial conversation. Immediately after their participation, dyad members evaluated the interaction along a number of qualitative dimensions, such as conflict, influence, enjoyment, disclosure, and intimacy. Thus, participants provided on-line evaluations of their encounters. In addition, observers who were unacquainted with the participants later viewed and provided evaluations of the videotaped interactions. Thus, the relations of peoples' levels of positive and negative affect to independent observers' ratings of their interactions, as well as to their own perceptions of them, could be examined. 
In Study 2 we used interaction diaries to obtain measures of the quantity and quality of people's daily social encounters. Specifically, 105 participants recorded all interactions of 10 min or longer in which they participated during a 1-week period. The records were completed as soon as possible after each interaction ended. In addition to providing information about interaction frequency, length, and the partners involved, participants evaluated each interaction along a variety of qualitative dimensions, such as intimacy, disclosure, purpose, control, and pleasantness. Thus, information about NA, PA, and their relations to the quantity and quality of various types of naturally occurring interactions (e.g., groups, opposite-sex dyads) could be assessed. Moreover, because participants provided their descriptions and evaluations of specific interactions shortly after they occurred, potential problems with biased recall were again circumvented. In sum, these studies together provide a view of the relations between affect and interaction that is much more detailed, and likely more accurate, than previously available.

\section{Study 1}

\section{Method}

Participants. One hundred twelve undergraduate women enrolled in Introductory Psychology at Southern Methodist University participated in Study I. The women were paired into 56 dyads. Dyad assignment was randomly determined, with the constraint that partners not be previously acquainted.

Procedure. Interested individuals signed up for an experiment on "group processes" in exchange for extra credit points. A research assistant (RA) randomly divided the women into pairs and contacted each potential participant by telephone to schedule an experimental session. Participants were informed of the name of their potential partner and were asked whether they were acquainted. If they were, that individual was assigned to a different partner. Because most of the participants were first-semester freshmen, we encountered few difficulties assigning people to unfamiliar dyad partners.

The experimental procedure used involved a modification of Ickes's unstructured dyadic interaction paradigm (e.g., Ickes, Bissonnette, Garcia, \& Stinson, 1990; Ickes, Robertson, Tooke, \& Teng, 1986; Ickes \& Tooke, 1988). Participants were greeted by the RA, taken to a medium-size room, and seated on a couch. A video camera was present, and the RA explained that part of the study involved the development of a set of videotapes for future studies of social perception. She then went to the camera, ejected the videotape, and indicated that the wrong tape had been left in the machine. The participants were asked to wait together while the RA left the laboratory, supposedly to retrieve the correct videotape. The pair was left alone for $6 \mathrm{~min}$. During that time, they were surreptitiously filmed by a second camera located in a darkened, adjoining equipment room that had been activated by remote control when the RA left. Although they had not been specifically directed to talk with one another, all dyads spontaneously engaged in conversation.

After returning, the RA placed the participants in different rooms and asked each to evaluate the interaction in which they had just participated. Specifically, dyad members indicated the extent to which they enjoyed the interaction; the extent to which the interaction was smooth, natural, and relaxed; how much they would like to interact with their partner again; how much their partner disclosed to them; how much they disclosed to their partner; the extent to which the interaction was forced, strained, and awkward; the extent to which they influenced the interaction; the extent to which their partner influenced the interaction; the extent to which the interaction was intimate; the extent to which the interaction was satisfying; and the extent to which the interaction was pleasant. These measures were obtained on 8-point scales with endpoints of not at all (1) and very much (8).

After completing a series of additional tasks that were unrelated to the present study, ${ }^{1}$ the participants were given a packet containing several self-report items, including the Positive and Negative Affect Schedule (PANAS; Watson, Clark, \& Tellegen, 1988), a self-report measure of PA and NA that has well-established psychometric properties. The PANAS consists of a list of 10 words that describe negative feelings or emotions (e.g., hostile, nervous) and 10 words that describe positive feelings or emotions (e.g., enthusiastic, proud). Respondents indicate on a 5-point scale "to what extent you generally feel this way, that is, how you feel on average." Responses are summed separately for the 10 positive words and the 10 negative words, yielding trait NA and trait PA scores that may each range from 10 to 50 . Participants were asked to complete the self-report items within 2 weeks and to return the packet to the psychology department.

Two observers who were unacquainted with the participants also provided their impressions of the 56 videotaped interactions. The observers independently viewed the social encounters and rated each on four dimensions. These included the extent to which (a) the partners seemed to enjoy the interaction, (b) the partners seemed engaged and involved in the interaction, (c) the interaction seemed intimate, and (d) the partners disclosed personal information to one another. These evaluations were made on bipolar scales with endpoints of not at all (1) and very much (8).

\section{Results}

Positive and Negative Affect scores. Our participants' PA scores ranged from 16 to $50(M=36.2, S D=6.62)$. Their NA scores ranged from 10 to $36(M=20.5, S D=6.00)$. These values are very similar to those observed in other samples (e.g., Watson et al., 1988). A moderate significant negative correlation between participants' NA and PA scores was revealed, $r(110)=-.27, p<.05$. Again, the direction and magnitude of this relation is consistent with those observed in previous studies (e.g., Watson, 1988a, 1988b). Because participants had been randomly assigned to dyad partners, one would expect dyad members' affect scores to be uncorrelated. Dyad members' levels of NA were indeed unrelated (intraclass $r=.17, n s$ ), as were their PA scores (intraclass $r=-.10, n s$ ).

Participants' social interaction judgments. Recall that participants had evaluated their interactions on 11 qualitative dimensions. We first examined the relations between dyad members' ratings of their shared interaction. As one would expect, peoples' evaluations of their interactions were generally influenced by their partners' views. Significant intraclass correlations were revealed between both dyad members' ratings of the extent to which their encounters were enjoyable $(.34)$, relaxed (.33), pleasant (.38), and satisfying (.27); how much they liked each other $(.36)$; and how much they would like to interact again $(.28$, all $p \mathrm{~s}<.05)$. The relations between dyad partners' judgments of how intimate and how awkward the interaction seemed, the extent to which each participant influenced the interaction, and the extent to which partners self-disclosed did not attain significance (all $p s>.05$ ).

\footnotetext{
${ }^{1}$ The additional tasks all took place after the events described here. They pertained to an unrelated study of physical appearance, nonverbal style, and leadership emergence.
} 
Next, we used factor analysis to determine whether the 11 original scales on which participants had evaluated their interactions might be reduced to a more manageable number of dimensions. A varimax rotation revealed three factors. The first (factor loadings appear in parentheses) incorporated ratings of enjoyment (.84), smooth-natural-relaxed (.87), desire to interact again $(.75)$, forced-strained-awkward $(-.70)$, pleasantness $(.80)$, and satisfaction with the interaction ( .80$)$. We averaged these ratings to create a Quality composite. The second factor incorporated the ratings of the extent to which the participants self-disclosed (.93) and the extent to which they felt their partners had self-disclosed (.91). The mean of these ratings comprised a Disclosure composite. The extent to which participants felt that they $(.85)$ and their partners $(.81)$ influenced the interaction loaded on the third factor, and these ratings were averaged to create an Engagement dimension. Finally, ratings of the intimacy of the interaction loaded substantially on both the Quality (.40) and Disclosure (.57) factors. Thus, this scale was treated separately in further analyses. Where necessary, scales were reversed so that high scores on these dimensions indeed reflected high levels of quality, disclosure, engagement, and intimacy.

Did participants' positive and negative affect scores predict their own and their partners' evaluations of the interactions? Following the recommendations of other researchers (e.g., Ickes et al., 1986; Kenny \& LaVoie, 1985), we computed the relations between affect scores and participants' judgments of interaction quality at both the dyad level and at the adjusted individual level. The dyad-level analyses examined whether a dyad's mean level of either positive or negative affect predicted the average of the two members' evaluations of the interaction along a given dimension. We calculated two sets of adjusted individual-level analyses. The first examined the relations between a person's level of PA and NA and her own ratings of the interaction. The second set of adjusted individual-level analyses examined the extent to which a person's levels of PA and NA predicted her partner's ratings of the interaction. ${ }^{2}$ Finally, parallel sets of dyad-level and adjusted individual-level partial correlations assessed the relations between PA and evaluations of the interactions controlling for NA, and the relations between NA and evaluations of the interactions controlling for PA. All are displayed in Table 1.

Positive affect was a consistent predictor of the general quality of people's social interactions at the dyad level. Moreover, the adjusted individual-level analyses revealed that participants' PA levels were positively and significantly related to both self and partner ratings of the quality of the interactions in which they engaged. PA was unrelated to evaluations of the amount of disclosure, engagement, or intimacy that characterized the interactions. Negative affect did not predict evaluations of the quality, engagement, or intimacy of the interactions in which people engaged. However, the adjusted individual analyses revealed that NA was marginally related to the amount of self-disclosure in which people reported that they engaged during these encounters.

Observers' social interaction judgments. Two independent observers had viewed the videotaped social interactions and provided ratings of their pleasantness, disclosure, engagement, and intimacy. Agreement between the two judges' ratings of the
56 interactions on each were as follows: pleasantness: $r=.62$, disclosure: $r=.55$, engagement: $r=.74$, and intimacy: $r=.61$ (all $p s<.01$ ). We computed the mean of the two observers' ratings of each interaction along each of the four scales for use in further analyses. We then examined the interrelations among these four rating scales. Recall that participants' evaluations of the levels of enjoyment, disclosure, intimacy, and engagement that characterized the interactions in which they took part comprised four different dimensions. However, the ratings along these four scales provided by our observers were all very highly correlated (mean $r=.86$ ). Thus, observers who viewed the videotaped interactions simply discriminated them along one general dimension of positive versus negative, whereas the evaluations of the people who actually participated in those interactions were multidimensional and more complex. Because judges' ratings along the four individual scales examined were so similar, we computed the mean of the four ratings obtained for each interaction. This composite variable reflected observer judgments of the general overall quality of the social interactions.

Did participants' positive and negative affect scores predict independent observers' evaluations of the interactions? We examined the relations between participants' levels of affect and observers' evaluations of the interaction. Specifically, the mean of the PA scores observed for each member of a dyad was correlated with the rated quality of their interaction, as was the average of their NA scores. In addition, we computed partial correlations that assessed the relations of PA to observer judgments controlling for NA and assessed the relations of NA to observer judgments controlling for PA. As can be seen in Table 1, interactions involving high-PA individuals were evaluated more positively by independent observers than were interactions in which low-PA participants took part. Moreover, partial correlations revealed that these effects were independent of participants' levels of negative affect. Participants' levels of negative affect were unrelated to observer ratings of their interactions. Thus, although independent judges could discriminate interactions in-

\footnotetext{
${ }^{2}$ The specifics of these analytical approaches are described here for interested readers. The dyad-level analyses treat the dyad as the unit of analysis. For each dyad, we calculated the average of both members' PA scores, NA scores, and ratings on each evaluation scale. The dyad-level (mean) PA scores were then correlated with the dyad-level (mean) evaluation scores, as were the dyad-level (mean) NA scores. The adjusted level analyses focus on the individual-level variance that remains when dyad variance is controlled. We carried out adjusted individual-level analyses of the relations between participants" affect scores and their own evaluations of the interaction by (a) calculating the deviation of each individual's own affect score from the dyad's mean affect score, (b) calculating the deviation of each individual's own evaluation score from the dyad's mean evaluation score, and (c) correlating the two deviation scores. We carried out adjusted individual-level analyses of the relations between participants' own affect scores and their partner's evaluations of the interaction by (a) calculating the deviation of each participant's own affect score from the dyad's mean affect score, (b) calculating the deviation of each participant's partner's evaluation score from the dyad's mean evaluation score, and (c) correlating the two deviation scores ( see Kenny \& LaVoie, 1985, and Ickes et al., 1986, for details regarding the statistical foundations of these approaches to analyzing interdependent dyadic data).
} 
Table 1

Relations Between Participants' Positive Affect (PA), Negative Affect (NA), and

Self, Partner, and Observer Ratings of Interaction

\begin{tabular}{|c|c|c|c|c|c|c|c|c|}
\hline \multirow[b]{3}{*}{$\begin{array}{l}\text { Dependent } \\
\text { measure }\end{array}$} & \multicolumn{6}{|c|}{ Participant ratings } & & \\
\hline & \multicolumn{2}{|c|}{ Dyad ratings ${ }^{\mathbf{a}}$} & \multicolumn{2}{|c|}{ Self ratings ${ }^{b}$} & \multicolumn{2}{|c|}{ Partner ratings ${ }^{\mathbf{b}}$} & \multicolumn{2}{|c|}{ Observer ratings $^{\mathrm{a}}$} \\
\hline & $\begin{array}{l}\text { Zero- } \\
\text { order } r\end{array}$ & $\begin{array}{c}\text { Partial } \\
r\end{array}$ & $\begin{array}{l}\text { Zero- } \\
\text { order } r\end{array}$ & $\begin{array}{c}\text { Partial } \\
r\end{array}$ & $\begin{array}{l}\text { Zero- } \\
\text { order } r\end{array}$ & $\begin{array}{c}\text { Partial } \\
r\end{array}$ & $\begin{array}{l}\text { Zero- } \\
\text { order } r\end{array}$ & $\begin{array}{c}\text { Partial } \\
r \\
\end{array}$ \\
\hline \multicolumn{9}{|c|}{ Positive affect ${ }^{\mathfrak{c}}$} \\
\hline $\begin{array}{l}\text { Quality } \\
\text { Disclosure } \\
\text { Engagement } \\
\text { Intimacy } \\
\end{array}$ & $\begin{array}{r}.32^{*} \\
.03 \\
-.07 \\
-.09 \\
\end{array}$ & $\begin{array}{r}.31^{*} \\
.10 \\
-.02 \\
-.04 \\
\end{array}$ & $\begin{array}{l}.27^{*} \\
.06 \\
.10 \\
.09 \\
\end{array}$ & $\begin{array}{l}.24^{e} \\
.11 \\
.10 \\
.09 \\
\end{array}$ & $\begin{array}{l}.29^{*} \\
-.11 \\
-.09 \\
-.08 \\
\end{array}$ & $\begin{array}{l}.28^{*} \\
-.09 \\
-.07 \\
-.10 \\
\end{array}$ & $.38^{* *}$ & $.34^{* *}$ \\
\hline \multicolumn{9}{|c|}{ Negative affect ${ }^{d}$} \\
\hline $\begin{array}{l}\text { Quality } \\
\text { Disclosure } \\
\text { Engagement } \\
\text { Intimacy }\end{array}$ & $\begin{array}{r}-.11 \\
.17 \\
.17 \\
.14\end{array}$ & $\begin{array}{r}-.07 \\
.20 \\
.15 \\
.11\end{array}$ & $\begin{array}{l}-.16 \\
.23^{e} \\
-.05 \\
-.01\end{array}$ & $\begin{array}{c}-.14 \\
.23^{\mathrm{e}} \\
-.03 \\
.01\end{array}$ & $\begin{array}{r}.16 \\
-.20 \\
.03 \\
-.01\end{array}$ & $\begin{array}{r}.15 \\
-.20 \\
.02 \\
-.01\end{array}$ & -.12 & -.09 \\
\hline
\end{tabular}

Note. $\quad N$ of participants $=112 ; N$ of dyads $=56$. For dyad-level analyses, $d f=54(N$ dyads -2$)$; for adjusted individual-level $\mathrm{s}, d f=55(N$ dyads -1$)$. Delete an additional $d f$ for the partial $r s$.

${ }^{a}$ Dyad level. ${ }^{\mathrm{b}}$ Adjusted-individual level. ${ }^{\mathrm{c}}$ Partial correlations control for NA. " Partial correlations control for PA. ' Marginally significant at $p<.07$.

${ }^{*} p<.05 .{ }^{* *} p<.01$.

volving dyads high in PA from those involving dyads low in PA, observers could not as readily discern the interactions engaged in by high-NA people from those engaged in by low-NA people. ${ }^{3}$

\section{Summary}

The results of Study 1 reveal that people's levels of positive affect predict qualitative aspects of their social experiences. In particular, high-PA individuals perceive their own interactions to be more enjoyable, comfortable, and pleasant than do lowPA people. Moreover, participants' levels of positive affect predicted their partner's evaluations as well as impressions of their videotaped interactions provided by judges who were not acquainted with them. In particular, the interactions in which high-PA participants engaged were perceived to be of a higher general quality than were those that involved low-PA participants. In addition, people who are high in negative affect tend to view their own interactions as involving higher levels of disclosure than do people who are low in NA. However, observers did not seem to differentiate high- and low-NA individuals' interactions along the dimensions assessed.

\section{Study 2}

In Study 1 , participants engaged in initial conversations with partners to whom they had been randomly assigned. In Study 2 , individuals kept records of the frequency, nature, and quality of their daily social encounters. Thus, the data collected in Study 2 differ from and complement those featured in Study 1 in several ways. In Study 1 we examined one dyadic interaction per participant, which took place under standardized conditions, whereas Study 2 features data from numerous reports of naturally occurring interactions. A number of advantages accrue from the control gained in Study 1; we have information about both dyad members' levels of NA and PA, we have data regarding both partners' experiences, and we were able to obtain objective (i.e., observer) ratings of the interactions. However, the more naturalistic approach taken in Study 2 has its own advantages. For example, we can obtain information about daily interaction frequency as well as quality, and information about various types of interactions is available. Thus, the data from Study 2 allow us to assess whether the relations between NA and PA and qualitative aspects of interactions revealed in Study 1 generalize to people's descriptions of their naturally occurring interactions and to examine the relations of PA and NA to both the quantity and quality of different types of interactions.

\section{Method}

Participants. The Study 2 sample involved 105 people who participated in a recent study of the relations between physical appearance and social interaction (Berry \& Landry, 1996). These students ( 43 men and 62 women) kept diaries of their social interactions for 1 week. ${ }^{4}$ Each received pass/fail independent study credit for their assistance. None of

\footnotetext{
${ }^{3}$ We also examined the correlations between PA, NA, and the four original observer rating scales. As one might expect, PA was positively related to judgments along all four dimensions, and NA was not related to any of these judgments.

${ }^{4}$ Actually, a total of 121 participants maintained interaction diaries. However, only 114 completed the experiment, and PANAS scores were available for only 105 of these participants. All data reported in this article are based on this final sample of 105 .
} 
the participants had taken part in Study 1. First-year students composed $12 \%$ of the sample, $38 \%$ were second-year students, $30 \%$ were juniors, $15 \%$ were seniors, and 5\% were graduate students. Eight percent of the participants reported that they lived with their parents, $28 \%$ lived in campus dormitories, and $64 \%$ lived in a house or apartment but not with their parents. Individuals taking part in the study were allowed to select the particular week of the semester during which they participated, with the exception of the first or last week of classes, finals week, or any week containing a university holiday.

Social interaction diaries. The diaries maintained by the participants were modified versions of the Rochester Interaction Record, which has been used in a number of studies of social interaction (e.g., Nezlek, Imbrie, \& Shean, 1994; Reis et al., 1982; see Reis \& Wheeler, 1991, for a review). In the current study, sets of 20 individual interaction records were bound into $4 \times 5$-in. booklets that were small enough for participants to conveniently carry with them during the recording period. People were instructed to record their interactions as soon after they occurred as possible. Participants wrote their subject code number on each record, as well as the date, time, and length of the interaction. They provided demographic information about their partners in the interaction (e.g., number, sex). For dyadic interactions, participants were asked to indicate whether their partner was someone with whom they were unacquainted, someone with whom they were acquainted but not close, or someone who was a close acquaintance. Each interaction was then described on a series of 8-point scales that inquired about the extent to which the task involved work versus recreation, was intimate, involved self-disclosure, involved disclosure from the partner(s), was initiated by the partner(s), was influenced by the partner(s), involved conflict, and was pleasant. A sample interaction record is displayed in Figure 1.

Procedure. Individuals who indicated interest in the project were contacted by phone and scheduled for a preliminary interview, during which a detailed description of what participation in the study would involve was provided. We encouraged people to take part only if they felt comfortable with the procedures described and if they were reasonably certain that they would be able to complete the entire week of record keeping. In line with previous studies (cf. Reis \& Wheeler, 1991), we did not refer to the participants as the "subjects" of the study. Rather, their role was described as one of "research assistant". The goal of their participation was described as the accumulation of a large database of observations about patterns of daily social interaction.

Following previous research, participants were instructed to keep records of each of their interactions that lasted $10 \mathrm{~min}$ or longer. The following definition of an interaction, adapted from previous studies (e.g., Kashy, 1991, p. 22; Wheeler \& Nezlek, 1977, pp. 743-744), was provided:

An interaction occurs when you and at least one other person pay attention to one another and adjust your behavior to one another. This study is concerned only with interactions that last at least 10 minutes or longer in duration. A conversation is the clearest example of an interaction. Person A says something. Person B responds, and so forth. Dancing and playing games are also interactions. Sitting side by side and watching television is not an interaction unless you are having a running dialogue while the television is on. Listening to a lecture is not an interaction, even if you occasionally ask a question. However, a class discussion group would be an interaction. Let's take a more difficult example. Suppose you are dining with a group of people. You are listening to their conversation but seldom say anything yourself. Do you record this as an interaction? It is a social interaction if you are following the conversation and could enter into it if you wished. If you are not following the conversation, or if it would be inappropriate for you to enter it, it would not be recorded as a social interaction. Let's take another difficult example. If you interacted with Person $A$ for an hour, but you are joined by Person $B$ for 20 minutes during the middle third of the hour, three interactions should be recorded: A 20-minute interaction with Person A, a 20-minute interaction with both $A$ and $B$, and a 20-minute interaction with $A$.

Participants were given an interaction record to look over and were encouraged to ask questions about the diaries or any other aspect of the procedure. They were also given the phone number of the experimenter and were encouraged to call for guidance if they had questions, concerns, or problems at any time during the recording period. Very few difficulties in understanding the instructions or completing the record keeping task were reported.

People taking part in the study recorded their interactions for a 7 day period that began on a Sunday morning and ended the following Saturday night. At the preliminary meeting, participants were given a supply of record booklets that they began to use on the following Sunday. On Monday morning, they returned all completed booklets to the experimenter and picked up a fresh supply. Participants continued to turn in their diaries and meet with the experimenter on a daily basis, with one exception. It was not possible to schedule meetings with most individuals during the weekends. Because of this, participants picked up a sufficient number of booklets on Friday morning to use on both Friday and Saturday and returned all on the following Monday.

After completing the interaction diaries, each participant was scheduled for an individual postexperimental interview. During this interview, they anonymously completed a questionnaire about the accuracy of their diaries. Specifically, they indicated on 8-point scales (a) how accurate they believed their own records to be, (b) the difficulty involved in recording their interactions, (c) their estimate of how accurate the diaries of other participants in the study were, and (d) the extent to which the record keeping had interfered with their lives. They also estimated the proportion of their interactions that they had failed to report. These questions were adapted from previous studies in which similar methodologies were used (cf. Reis \& Wheeler, 1991).

Finally, participants received a take-home packet that contained several self-report items. In addition to the PANAS, we included the NEOFive Factor Inventory (NEO-FFI; Costa \& McCrae, 1985) in these packets. This measure assesses the dimensions of Extraversion, Neuroticism, Openness to Experience, Conscientiousness, and Agreeableness. As noted previously, PA and Extraversion have been proposed to reflect the same underlying dimension, as have NA and Neuroticism (Watson et al., 1992; Watson \& Clark, 1992). Moreover, previous work has revealed that the relations between the NEO scales of Extraversion and Neuroticism and participants' retrospective accounts of social activity parallel those of PA and NA, respectively (Watson et al., 1992). Therefore, we wanted to further examine the relations of these self-report items to the diary measures of social interaction. Participants were instructed to complete the contents of the packet within 2 weeks and return it to the psychology department.

\section{Results}

Distribution of personality scores. Our participants' PA scores ranged from 14 to $48(M=36.8, S D=6.00)$, and their NA scores ranged from 11 to $48(M=20.5, S D=6.29)$. The correlation between people's PA and NA scores was $r(103)=$ $-.35, p<.01$. This value is, again, very similar to those observed in previous studies. Participants' scores on the Extraversion scale of the NEO-FFI ranged from 27 to $58(M=44, S D=$ $6.49)$, and Neuroticism scores ranged from 17 to $59(M=34.2$, $S D=8.38)$. NEO Openness to Experience scores ranged from 27 to $57(M=42.1, S D=6.22)$. The mean of the observed Conscientiousness scores was 44.4 ( range $=20-58, S D=7.60$ ), 
Date Time am/pm Length of interaction: hours minutes

For each partner in the interaction:

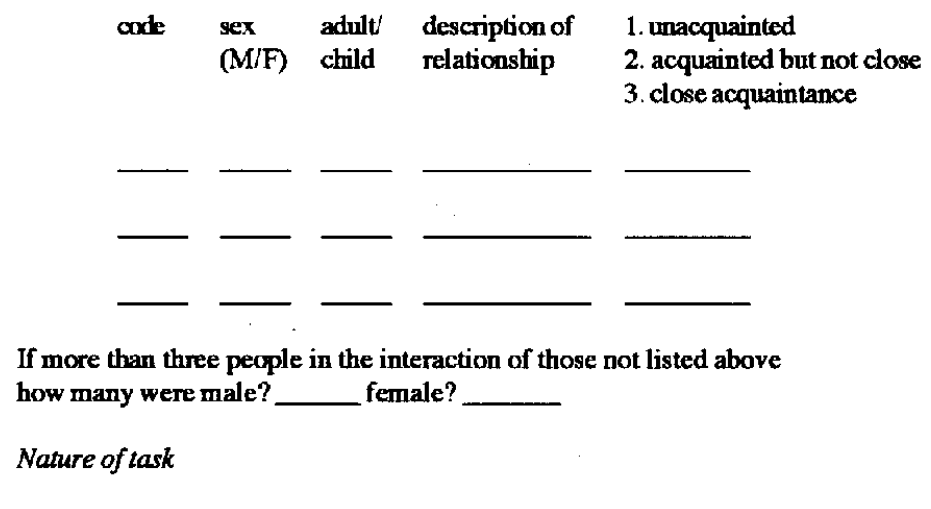

$\begin{array}{llllllllll}\text { Work oriented } & 1 & 2 & 3 & 4 & 5 & 6 & 7 & 8 & \text { Recreation oriented }\end{array}$

\begin{tabular}{|c|c|c|c|c|c|c|c|c|c|c|}
\hline About the interaction: & & & & & & & & & & \\
\hline Intimacy & superficial & 1 & 2 & 3 & 4 & 5 & 6 & 7 & 8 & meaningful \\
\hline Idisclosed & very little & 1 & 2 & 3 & 4 & 5 & 6 & 7 & 8 & a great deal \\
\hline Otherdisclosed & very little & 1 & 2 & 3 & 4 & 5 & 6 & 7 & 8 & a great deal \\
\hline Conflict & very little & 1 & 2 & 3 & 4 & 5 & 6 & 7 & 8 & a great deal \\
\hline Quality & unpleasant & 1 & 2 & 3 & 4 & 5 & 6 & 7 & 8 & pleasant \\
\hline Initiation & I initiated & 1 & 2 & 3 & 4 & 5 & 6 & 7 & 8 & other(s) initiated \\
\hline Influence & $\begin{array}{l}\text { Iinfluenced } \\
\text { more }\end{array}$ & 1 & 2 & .3 & 4 & 5 & 6 & 7 & 8 & $\begin{array}{l}\text { other(s) influenced } \\
\text { more }\end{array}$ \\
\hline
\end{tabular}

Figure 1. Sample social interaction record.

and scores on the Agreeableness scale ranged from 27 to 57 ( $M$ $=43.2, S D=6.53$ ).

Relations among personality scores. A varimax rotation yielded the five-factor solution depicted in Table 2. Consistent with previous research, positive affect and Extraversion loaded on one factor, and negative affect and Neuroticism scores loaded on a second. Openness to Experience, Conscientiousness, and Agreeableness each comprised individual factors. On the basis of these data, we combined positive affect and Extraversion scores to create a composite PA-Extraversion variable. Specifically, we converted PA scores and Extraversion scores to $z$ scores. The mean of the PA and Extraversion $z$ scores was calculated; this served as an index of PA-Extraversion. For the sake of simplicity, these values are referred to as positive affect scores throughout the remainder of this article. Negative affect scores and Neuroticism scores were combined in a like manner and 
Table 2

Factor Loadings of Personality Measures

\begin{tabular}{lccccr}
\hline \multicolumn{1}{c}{ Personality measure } & Factor 1 & Factor 2 & Factor 3 & Factor 4 & Factor 5 \\
\hline PANAS Positive Affect & $\mathbf{8 1}$ & -.30 & .07 & .32 & -.01 \\
NEO Extraversion & .85 & -.08 & .01 & -.19 & .31 \\
PANAS Negative Affect & -.06 & .93 & .01 & -.09 & -.09 \\
NEO Neuroticism & -.29 & .83 & .01 & -.18 & -.13 \\
NEO Openness to Experience & .04 & .01 & .99 & -.06 & .04 \\
NEO Conscientiousness & .03 & -.18 & -.07 & .95 & .11 \\
NEO Agreeableness & .19 & -.17 & .04 & .12 & .94 \\
\hline
\end{tabular}

Note. $\quad$ PANAS $=$ Positive and Negative Affect Schedule; NEO $=$ NEO Personality Inventory. $N=105$. Factor loadings of higher magnitude than .40 are shown in boldface type.

are simply referred to as negative affect scores. Participants' scores on these two composite variables, along with their Openness to Experience, Conscientiousness, and Agreeableness scores, served as predictors of the social interaction indices derived from their diaries.

Self-judged accuracy of social interaction diaries. During the follow-up interview, participants answered a series of questions about the accuracy of their interaction diaries. As can be seen in Table 3, people judged their records to be quite accurate and indicated that participation in the study was neither difficult nor especially disruptive of their daily routines. These data are similar to those obtained by other researchers who have used social interaction diaries. For example, Reis and Wheeler (1991) reported that individuals in several of their studies estimated that they had failed to report an average of $7.2 \%$ of their interactions, whereas participants in our study guessed that they had failed to record an average of $8.2 \%$ of their interactions.

Database. The 105 participants together provided descriptions of 4,781 social interactions. We used these to create a data file that contained a variety of quantitative and qualitative indices generated for each individual person. The quantitative measures included the raw number of interactions, the mean length of interactions, and the total amount of time during the recording period spent engaged in interaction reported by each participant. We calculated these values separately for dyadic oppositesex interactions, dyadic same-sex interactions, and group interactions, as well as for all interactions reported by a given

Table 3

Self-Reported Accuracy of Social Interaction Diaries

\begin{tabular}{lll}
\hline \multicolumn{1}{c}{ Dependent measure } & $M$ & $S D$ \\
\hline $\begin{array}{l}\text { Difficulty of recording interactions (not at all } \\
\text { difficult-very difficult) }\end{array}$ & 2.46 & 1.35 \\
$\begin{array}{l}\text { Accuracy of own records (very accurate-not at all } \\
\text { accurate) }\end{array}$ & 2.66 & 1.39 \\
$\begin{array}{l}\text { Accuracy of other participants' records (very } \\
\begin{array}{l}\text { accurate-not at all accurate) } \\
\text { Record keeping interfered with normal life (not at }\end{array}\end{array}$ & 3.33 & 1.25 \\
\begin{tabular}{l} 
all-a substantial amount) \\
\hline
\end{tabular}
\end{tabular}

Note. $N=105$, Questions were answered on bipolar scales with endpoints of 1 and 8 . individual. In addition, we calculated the proportion of each person's total interactions that fell into these three individual categories.

Participants had originally been asked to provide qualitative descriptions of their interactions on eight different scales. A factor analysis was performed to determine whether these descriptions could be reduced to a smaller number of underlying dimensions. Specifically, for a particular qualitative dimension, we calculated the mean score observed for a given participant, collapsing across all of their individual interactions. We carried out this procedure for each of the eight scales and subjected the resulting values to a varimax rotation. Three factors emerged from this analysis. The first incorporated ratings (factor loadings appear in parentheses) of "I disclosed", "other disclosed", and "intimacy" $(.92, .91$, and .67 , respectively). The ratings of "quality" and "conflict" loaded on the second factor (.89 and -.82 ), and ratings of the extent to which the "other(s) influenced" and "other(s) initiated" the interaction loaded on the third factor (.77 and .79). Finally, the scale on which participants described their interactions as task versus recreation did not clearly load on any of the three factors just described and was therefore treated separately in further analyses. ${ }^{5}$

On the basis of this information, we constructed four final measures from the original eight descriptors of interaction quality. Evaluations of the extent to which "I disclosed", "other disclosed", and "intimacy" were averaged to create a Disclosure composite. Ratings of interaction "quality" and "conflict" (pole reversed) together composed an Enjoyment composite. Evaluations of the extent to which the "other(s) initiated" and "other(s) influenced" were averaged (poles reversed) to form a Control dimension. Finally, the scale on which individuals evaluated the nature of each interaction (task vs. recreation) was analyzed individually and is referred to as a Recreation dimension. The mean of each participant's evaluations of their total interactions along each of these four dimensions was included in the database. In addition, we calculated the mean evaluation along each dimension that people gave to their dyadic oppositesex interactions, dyadic same-sex interactions, and group interactions. Finally, we calculated the mean closeness rating pro-

\footnotetext{
${ }^{5}$ We should note that we also performed a factor analysis on the eight ratings of the 4,781 individual interactions. A similar pattern of results emerged from both analyses.
} 
Table 4

Sample Means of Quantitative Measures of Social Interaction

\begin{tabular}{lcccc}
\hline Quantitative measure & $\begin{array}{c}\text { All } \\
\text { interactions }\end{array}$ & $\begin{array}{c}\text { Opposite- } \\
\text { sex dyads }\end{array}$ & $\begin{array}{c}\text { Same- } \\
\text { sex dyads }\end{array}$ & $\begin{array}{c}\text { Group } \\
\text { interactions }\end{array}$ \\
\hline Number & 45.5 & 13.8 & 17.8 & 13.7 \\
Length (in minutes) & 55.9 & 60.1 & 41.6 & 77.9 \\
Total time (in minutes) & 2,448 & 804 & 725 & .39 \\
Proportion & & .32 & .361 \\
\hline
\end{tabular}

Note. $\quad N=105$. Number $=$ raw number of reported interactions in each category; $L$ ength $=$ average duration of interactions reported in each category; Total time = total amount of time during reporting period spent engaged in each type of interaction; Proportion = proportion of total interactions attributable to each category.

vided by participants for their partners in opposite-sex dyadic and same-sex dyadic interactions. The means of the major social interaction indices appear in Table 4.

Overall quantity of social interaction. As can be seen in Table 4 , people reported engaging in an average of 45 social interactions during the 7 -day recording period. The average duration of these interactions was $56 \mathrm{~min}$ (recall that only interactions of $10 \mathrm{~min}$ or longer were recorded). Thus, our participants spent approximately $41 \mathrm{~h}$ per week involved in such social activity. On average, $39 \%$ of these interactions involved one samesex partner, 32\% involved one opposite-sex partner, and $29 \%$ were group interactions. People tended to be very well acquainted with those individuals with whom they spent time. Seventy-five percent of the dyadic interactions recorded were described by participants as involving someone to whom they were close, $21 \%$ involved an acquaintance who was not close, and only $4 \%$ involved someone with whom they were previously unacquainted. These percentages were virtually identical for same-sex and opposite-sex interactions.

Overview of analyses. To examine the relations between dimensions of personality and social interaction, we calculated a series of multiple regression equations in which participants' positive affect and negative affect composite scores and their Openness to Experience, Conscientiousness, and Agreeableness scores were simultaneously entered as predictors of each quantitative and qualitative measure of social interaction. We first discuss the results obtained for all social interactions. We then examine the analyses conducted on descriptions of the individual categories of opposite-sex dyadic interactions, same-sex dyadic interactions, and group interactions. Because our interest is specifically in the relations between PA and NA and social interaction, the results for the dimensions of Openness to Experience, Conscientiousness, and Agreeableness are not the focus of our discussion. However, the relations between those variables and the social interaction measures are displayed in the tables and are briefly summarized in the text for interested readers.

Relations between positive affect, negative affect, and quantity of overall daily interaction. Table 5 displays the standardized beta weights obtained from three regression equations in which participants' NA, PA, Openness to Experience, Conscientiousness, and Agreeableness scores were simultaneously entered as predictors of their total number of interactions, the average length of their interactions, and the total amount of time during the recording period that they spent engaged in social activity. (All effects discussed in the text have betas that differ from zero at $p<.05$, unless otherwise noted.) ${ }^{6}$ Previous studies featuring retrospective estimates have found that $\mathrm{PA}$ is associated with frequency of and time spent in social contact (e.g., Watson, 1988a; Watson et al., 1992). Consistent with these reports, we found a significant positive relation between PA and on-line records of the total amount of time during which our participants engaged in social activity, and between the total number of interactions in which they engaged during the recording period and their level of trait PA. Unlike the majority of those previous studies, however, we also observed a significant relation between NA and the number of social encounters in which people engaged, as well as between NA and the total time that participants spent engaged in social contact. Thus, on-line recordings of social interactions suggest that high levels of either NA or PA are associated with increased overall affiliation. No significant relations were revealed between NA or PA and the average duration of people's social interactions.

Relations between positive affect, negative affect, and quality of overall daily interaction. Relations between participants' NA and PA scores and their mean on-line appraisals of their interactions along the dimensions of recreation, enjoyment, disclosure, and control also appear in Table 5. In a replication of the findings of Study 1, higher levels of PA were associated with reports of more enjoyable interactions. In addition, the interactions engaged in by people who were high in PA were more oriented toward recreation (vs. work) than were those experienced by low-PA individuals. NA was unrelated to people's overall evaluations of their social encounters.

Relations between additional NEO factors and quantity and quality of overall daily interaction. As can be seen in Table 5, the dimensions of Openness to Experience, Conscientiousness, and Agreeableness were unrelated to any of the quantitative measures of social interaction. However, Openness to Experience scores were negatively related to people's overall evaluations of the extent to which their interactions involved recreation and positively related to their judged level of disclosure. Conscientiousness scores were positively related to participants' evaluations of enjoyment and disclosure. Finally, Agreeableness

\footnotetext{
'Additional information regarding the regression analyses (zero-order correlations, means, standard deviations, etc.) may be obtained from Diane S. Berry.
} 
Table 5

Standardized Beta Weights From Multiple Regression Equations Predicting Overall Social Interaction Indices From Personality Dimensions

\begin{tabular}{|c|c|c|c|c|c|}
\hline Measure & $\begin{array}{c}\text { Positive affect- } \\
\text { Extraversion }\end{array}$ & $\begin{array}{l}\text { Negative affect- } \\
\text { Neuroticism }\end{array}$ & $\begin{array}{l}\text { Openness to } \\
\text { Experience }\end{array}$ & Conscientiousness & Agreeableness \\
\hline \multicolumn{6}{|l|}{ Quantitative } \\
\hline Number & $.34 * *$ & $.40^{* *}$ & .01 & .02 & .10 \\
\hline Mean length & -.04 & .08 & -.09 & -.05 & -.03 \\
\hline Total time & $.23^{*}$ & $.31^{* *}$ & -.03 & -.03 & -.01 \\
\hline \multicolumn{6}{|l|}{ Qualitative } \\
\hline Recreation & $.23^{*}$ & -.03 & $-.24^{* *}$ & -.05 & .18 \\
\hline Enjoyment & $.32^{* *}$ & .08 & -.01 & $.21^{*}$ & $.22^{*}$ \\
\hline Disclosure & -.05 & .11 & $.19^{*}$ & $.23^{*}$ & .17 \\
\hline Control & .03 & -.06 & -.05 & .15 & $-.25^{*}$ \\
\hline Partner closeness & .03 & -.11 & -.16 & .02 & -.01 \\
\hline
\end{tabular}

Note. $N=105$.

$* p<.05 . \quad * * p<.01$.

scores were related to the extent to which people reported enjoying and exerting control over their social encounters.

Relations between positive affect and quantitative and qualitative measures of individual categories of interactions. To get a better sense of what the observed relations between PA, NA, and measures of the participants' overall interactions reflect, we broke social encounters down into three different types and considered the relations of NA and PA to the quantity and quality of each. Specifically, we computed regression equations paralleling those just reported separately for opposite-sex dyadic interactions, same-sex dyadic interactions, and group interactions. We first discuss the relations observed between PA and characteristics of these three categories of interactions, which appear in Table 6. The overall relation between PA and interaction quantity appears to reflect a general tendency toward affiliation; high-PA individuals have greater numbers of group interactions and same-sex dyadic interactions than do low-PA individuals. The raw numbers of opposite-sex interactions reported by participants also bore a positive albeit nonsignificant relation to PA. High-PA individuals also engaged in significantly longer group interactions than did people with low levels of PA.

The overall relation between positive affect and interaction quality was also not particular to any one kind of interaction. Rather, people who were high in positive affect reported having more enjoyable opposite-sex dyadic interactions, better quality same-sex dyadic interactions, and higher quality group interactions than did people who were low in PA. Finally, high-PA individuals also reported that their same-sex dyadic interactions were more oriented toward recreational than work activities.

Relations between negative affect and quantitative and qualitative measures of individual categories of interaction. Recall that negative affect was also positively correlated with the overall frequency of people's interactions as well as with the amount of time they spent engaged in social interaction. As can be seen in Table 6 , this was mainly attributable to the number of samesex dyadic interactions in which high-NA people participated and the amount of time spent engaged in this type of interaction. In addition, as NA level increased, dyadic same-sex interactions made up increasingly larger proportions of people's total interactions. As this latter relation suggests, the previously described links between NA and amount of social contact did not reflect a general propensity of high-NA people to be more affiliative than low-NA people. In fact, as NA increased, the proportion of people's interactions that involved one member of the opposite sex decreased, and NA was not significantly related to the number of group interactions in which people engaged.

NA was also related to participants' qualitative evaluations of their social experiences. In particular, high-NA individuals" dyadic opposite-sex interactions were significantly less likely to be related to recreation (as opposed to work) than were low-NA people's interactions with members of the opposite sex. Moreover, high-NA participants reported being less close to their opposite-sex partners than did other participants. Unexpectedly, high-NA people reported more enjoyable group interactions than did low-NA people. High-NA people also reported greater levels of influence and less control over these group encounters than did other participants.

Relations between additional NEO factors and quantitative and qualitative measures of individual categories of interaction. Relations of the dimensions of Openness to Experience, Conscientiousness, and Agreeableness to various aspects of the individual categories of social interaction are also displayed in Table 6. Participants' Openness to Experience scores were positively related to the number of opposite-sex dyadic interactions that they experienced as well as the proportion of their total interactions that fell into this category. Openness to Experience scores were also positively related to the amount of disclosure that participants felt characterized their same-sex dyadic interactions. Conscientiousness scores predicted the extent to which people enjoyed their same-sex dyadic and group interactions. Participants' Agreeableness scores predicted the number of same-sex dyadic interactions they reported, as well as the proportion of their social encounters that were with individuals of the opposite sex. Agreeableness was also positively related to the extent to which people described their interactions with a member of the opposite sex as recreational and the extent to which their same-sex dyadic interactions were enjoyable, recreational in nature, and involved disclosure. Finally, Agreeableness was related to the amount of disclosure and control that characterized participants' group interactions. 


\section{Summary}

In sum, analyses of participants' social interaction diaries revealed that PA-Extraversion is positively related to the frequency and the pleasantness of people's interactions. This appears to be a general phenomenon and was not specific to any particular subtype of social encounter that we studied. The relations between NA-Neuroticism and social experience are somewhat more complex. First, NA is positively related to both overall interaction frequency and the total amount of time that people spend engaged in social contact. However, the relations observed between NA and interaction quantity do not reflect general gregariousness on the part of high-NA people. Rather, only the frequency of dyadic same-sex interactions was signifcantly correlated with NA. Moreover, these interactions made up greatex proportions of the social experiences of high-NA individuals than they did of the encounters described by low-NA people. In addition, dyadic opposite-sex interactions made up relatively small proportions of high-NA individuals' social lives, and high-NA participants described themselves as being less close to their opposite-sex partners than did other participants.

\section{General Discussion}

The data from Study 1 and Study 2 support previous findings regarding the relation between positive affect and interaction frequency, and they extend and clarify previous data regarding negative affect and quantity of social interaction. In addition, they provide new information about the relations of positive and negative affect to qualitative aspects of various kinds of social interactions, as evaluated by both the interaction participants and by independent observers. Below we summarize and discuss each of our primary findings.

\section{Positive Affect and Engagement in Social Activity}

A number of studies have noted a positive correlation between PA and self-reports of the amount of social activity in which people engage (e.g., Watson, 1988a; Watson et al., 1992). The social interaction diaries maintained by participants in Study 2 provide strong support for these findings. In particular, high-PA participants engaged in greater numbers of interactions and spent more total time involved in social activity than did low-PA individuals. A breakdown of participants' interactions by individual category revealed that the link between PA and overall amount of social activity generalized across different subtypes of social interactions.

\section{Positive Affect and Social Interaction Quality}

Study 1 and Study 2 provide clear and strong evidence that PA predicts the extent to which people feel that their interactions are pleasant, enjoyable, and satisfying. PA was positively related to self-reports of the quality of the interactions experienced by participants in Study 1, who evaluated an initial conversation with a randomly assigned partner, and by participants in Study 2, who provided records of their naturally occurring social contacts. Moreover, this relation was revealed for all categories of social interaction examined in Study 2. We should report that we have recently collected data that provide further 
support for this link. In this study (Berry, Meier, \& Pennebaker, 1996), previously unacquainted dyads participated in 1-h sessions designed to facilitate the development of interpersonal closeness through an induced self-disclosure procedure (Aron, Melanit, Aron, Vallone, \& Bator, 1995). At the end of the session, participants evaluated their experience. Although this procedure is quite successful in accelerating the development of intimacy, it is also rather intense and sometimes stressful for people. Even under these circumstances, participants' PA scores were positively related to their evaluations of how close they felt to their partner, how enjoyable and comfortable the procedure had been for them, and how much they would like to interact with their partner again. Thus, the link between PA affect and the extent to which people are generally satisfied with their interactions seems to be robust, and it generalizes across a wide range of contexts and circumstances.

In addition to predicting people's own descriptions of their social encounters, individuals' PA scores were related to how other people viewed their interactions. In particular, participants in Study 1 who were paired with high-PA partners evaluated their mutual interactions as more enjoyable than did participants paired with low-PA individuals. In addition, independent observers judged interactions in which high-PA participants were engaged to be of better quality than those that involved partners with lower PA scores. Thus, the social interactions of high-PA individuals differ from those of low-PA individuals in a manner that is readily identifiable by other people.

Can these findings shed any light on why PA is related to the extent to which people engage in social contact? Previous researchers have focused on explanations that posit a direct link between affect and social activity (e.g., Watson, 1988a; Watson et al., 1992). As we have noted, high-PA individuals may extract more enjoyment from social interaction than other people and, in turn, these positive feelings may reinforce their desire to engage in social contact (e.g., Cunningham, 1988a). The fact that our high-PA participants reported greater enjoyment of their interactions then did low-PA participants is entirely consistent with such an explanation of the PA-social activity link.

However, our data suggest that differences in the social constraints operating on high- and low-PA individuals may further contribute to the relation between PA and social engagement. As we noted previously, if high-PA people are perceived to be potentially engaging and interesting partners, others may be especially likely to provide them with opportunities for social contact. This explanation predicts that the interactions in which high-PA individuals engage will be "objectively" of high quality, in the sense that other people will perceive them to be preferable to other interactions. The fact that Study 1 participants' PA scores predicted the quality of their interactions as judged by both their partners, and by independent observers, provides strong support for this view. It should be noted that the two accounts of the PA-social activity link that we have discussed are by no means mutually exclusive, and our current studies were not intended to provide a critical test of these explanations. We do hope, however, that our data will encourage researchers to give greater consideration to social psychological factors when considering the source of relations observed between personality and social behavior.

\section{Negative Affect and Engagement in Social Activity}

An analysis of the interaction records generated in Study 2 reveals that negative affect is also positively related to the overall amount of social activity in which people engage. In particular, high-NA participants took part in greater numbers of interactions and spent more time involved in social contact than did low-NA participants. Previous studies have reported inconsistent relations between retrospective accounts of frequency of social activity and NA. Although some of these studies found NA and social contact to be unrelated (e.g., Watson, 1988a; Watson et al., 1992, Study 1), one did reveal a positive correlation between these variables (Watson et al., 1992, Study 2). Given these inconsistencies, replications of our current findings are certainly desirable. Note, however, that the study in which the link between NA and social activity was previously observed featured daily self-reports of social activity, whereas the others involved less frequent (e.g., weekly) reports. Because the data featured in that and the current studies are unlikely to be influenced by biased recall, we have reasonable confidence in this finding.

Why would NA be associated with increased affiliation? One possibility is that engaging in social activity increases one's level of negative affect. However, previous studies of within-subject variations in negative affect assessed before and after social interaction provide no evidence of this (McIntyre, Watson, \& Cunningham, 1990). Moreover, such an effect would seem to suggest that the social exchanges of high-NA individuals are characteristically unpleasant. Because participant, partner, and observer judgments of interaction quality were not related to trait NA in either of our studies, this explanation is rendered unlikely.

Reversing the causal arrows may provide a more plausible interpretation of this link: Perhaps high-NA people are predisposed to engage in social interaction, or at least in certain types of social interaction. It has long been recognized that negative emotions can lead to an increased desire for social contact to evaluate and interpret those feelings (e.g., Schachter, 1959). For example, people often feel compelled to talk about distressing events or feelings, and doing so can have very positive psychological effects (cf. Pennebaker, 1989). It may be the case that people who experience chronically high levels of NA spend a fair amount of time discussing and evaluating their feelings with other people as a method of coping with unpleasant emotions. Although our data do not allow us to test this hypothesis directly, the analyses of interaction frequency conducted within individual categories were generally consistent with such an interpretation. High-NA people did not experience elevated levels of all types of social contact; NA was unrelated to participation in group interactions, and high-NA individuals actually reported engaging in proportionally fewer opposite-sex dyadic interactions than did other participants. The positive overall relation between NA and affiliation reflected the propensity of highNA individuals to engage in extensive interactions with one same-sex individual. This may suggest that people who are high in NA spend a fair amount of time reflecting on their feelings and troubles with close friends. It should be noted that this positive relation between negative feelings and interaction would not be expected to hold for people experiencing extreme levels 
of psychological distress. For example, social withdrawal is a marker of clinical depression (e.g., Beck, 1967). Moreover, within depressed populations, severity of depressive symptomatology is negatively related to people's engagement in interactions with same-sex friends (Nezlek et al., 1994). However, for individuals drawn from nonclinical populations, seeking out this sort of social contact may constitute a useful strategy for dealing with NA. ${ }^{?}$

\section{Negative Affect and Social Interaction Quality}

This interpretation of the relation between NA and social engagement might further suggest that high-NA individuals' interactions would be characterized by relatively high levels of disclosure. However, we found few relations between NA and qualitative aspects of social experience in general, and only mixed support for a link between NA and disclosure in particular. In Study 1, a positive relation was revealed between participants' trait NA and self-reported disclosure in initial interactions with an unfamiliar same-sex partner. However, people's level of NA was not positively related to either their partner's or independent observers' estimates of the amount of disclosure that characterized these interactions. Moreover, NA was unrelated to self-reports of the amount of disclosure that characterized people's daily interactions in Study 2. A partial explanation of the latter discrepancy may involve differences in the extent of acquaintance of interaction partners in the two studies. In Study 1, our participants knew little about one another. On the other hand, the majority of interactions described by participants in Study 2 were with individuals they knew well. It may be that people use different baselines when evaluating the relative disclosure of their interactions with familiar and unfamiliar partners.

We have behavioral data from another study that are consistent with the relation between NA and self-judged disclosure suggested in Study 1. In a recent analysis of the linguistic correlates of positive and negative affect participants were videotaped while they talked to an interviewer with whom they were unacquainted (Berry, Pennebaker, \& Mueller, 1996). Text analyses revealed that high-NA people used more self-referents (e.g., $I$, $m e, m y s e l f)$ than did other people. Although not a direct measure of self-disclosure, that is consistent with the link between NA and self-reported disclosure observed in Study 1. At this point, these data need to be interpreted with caution, and this relation should be further explored in future research. ${ }^{8}$

\section{Conclusion}

A number of studies have examined the links between primary dimensions of personality, such as PA and NA, and other aspects of self-view. However, there has been much less attention to the relations between these basic dimensions of personality and more objective measures of social interaction and behavior, such as the observer ratings assessed in Study 1 and the on-line diary recordings of behavior featured in Study 2 . These studies contribute to a growing body of work that focuses on the interface between traditional approaches to the study of personality and the areas of social perception and social interaction (cf. Funder \& Colvin, 1991; Funder \& Sneed, 1993). In addition, the relations revealed between our measures of social experience and self-reports of PA and NA demonstrate the important role of personality in channeling social interaction. We hope that our data will encourage more researchers to pursue these important and interesting issues.

\footnotetext{
${ }^{7}$ In the study reported by Nezlek et al. (1994), social interaction diaries were used to compare the social experiences of depressed and nondepressed people. Although it is tempting to compare differences between these groups found by Nezlek et al, to the relations of NA and social activity reported here, NA and depression are very different constructs and would not necessarily be expected to relate to interaction patterns in a similar fashion. The relation between quantity of social engagement and these measures is a case in point.

${ }^{8}$ Some studies (e.g., Cunningham, 1988b) have reported a relation between positive moods and self-disclosure. However, that work focused on transient responses to mood induction rather than characteristic patterns of social behavior that may develop in response to traitlike variations in PA and NA assessed here.
}

\section{References}

Aron, A., Melanit, E., Aron, E. N., Vallone, R., \& Bator, R. (1995). The experimental generation of interpersonal closeness: $A$ procedure and some preliminary findings. Manuscript submitted for publication.

Beck, A. T. (1967). Depression: Clinical, experimental, and theoretical aspects. New York: Harper \& Row.

Berry, D. S., \& Landry, J. C. (1996). Social perception in the real world: Facial maturity and social interaction. Manuscript submitted for publication.

Berry, D. S., Meier, J. A., \& Pennebaker, J. W. (1996). Personality, linguistic, and nonverbal predictors of the development of interpersonal closeness. Manuscript in preparation, Southern Methodist University, Dallas.

Berry, D. S., \& Pennebaker, J. W. ( 1993). Verbal and nonverbal expression of emotion and health. Psychotherapy and Psychosomatics, 59, 11-19.

Berry, D. S., \& Pennebaker, J. W. (in press). Individual differences in nonverbal expression, inhibition, and bealth. In G. A. Fava \& $\mathbf{H}$. Freyberger (Eds.), Psychosomatic medicine. New York: Kraeger.

Berry, D. S., Pennebaker, J. W., \& Mueller, J. S. (1996). Emotions experienced and emotions expressed: Multiple dimensions of affect. Manuscript submitted for publication.

Conrath, D. W., Higgins, C. A., \& McClean, R. J. (1983). A comparison of the reliability of questionnaire versus diary data. Social Networks, 5, 315-322.

Cooper, H., Okamura, L., \& Gurka, V. (1992). Social activity and subjective well-being. Personality and Individual Differences, 13, 573583.

Costa, P. T., \& McCrae, R. R. (1980). Influence of extraversion and neuroticism on subjective well being: Happy and unhappy people. Journal of Personality and Social Psychology, 38, 668-678.

Costa, P. T., \& McCrae, R. R. (1985). The NEO Personality Inventory Mamual. Odessa, FL: Psychological Assessment Resources.

Cunningham, M. R. (1988a). What do you do when you're happy or blue? Mood, expectancies and behavioral interest. Motivation and Emotion, 12, 309-331.

Cunningham, M. R. (1988b). Does happiness mean friendliness? Induced mood and heterosexual self-disclosure. Personality and Social Psychology Bulletin, 14, 283-297.

Diener, E., \& Emmons, R. A. (1984). The independence of positive and negative affect. Journal of Personality and Social Psychology, 47, 1105-1117.

Emmons, R. A., \& Diener, E. (1985). Personality correlates of subjec- 
tive well-being. Personality and Social Psychology Bulletin, 11, 8997.

Forgas, J. P., Bower, G. H., \& Krantz, S. E. (1984). The influence of mood on perceptions of social interactions. Journal of Experimental Social Psychology, 20, 497-513.

Funder, D. C., \& Colvin, C. R. (1991). Explorations in behavioral consistency: Properties of persons, situations, and behaviors. Journal of Personality and Social Psychology, 60, 773-794.

Funder, D. C., \& Sneed, C. D. (1993). Behavioral manifestations of personality: An ecological approach to judgmental accuracy. Journal of Personality and Social Psychology, 64, 479-490.

Hodgins, H., \& Zuckerman, M. (1990). The effect of nonverbal sensitivity on social interaction. Journal of Nonverbal Behovior. 14, 155170.

Ickes, W., Bissonnette, V., Garcia, S., \& Stinson, L. (1990). Using and implementing the dyadic interaction paradigm. In C. Hendrick \& $\mathbf{M}$. Clark (Eds.), Review of personality and social psychology (Vol. 11, pp. 16-44). Beverly Hills, CA: Sage.

Ickes, W., Robertson, E., Tooke, W., \& Teng, G. (1986). Naturalistic social cognition: Methodology, assessment, and validation. Journal of Personality and Social Psychology, 51, 66-82.

Ickes, W., \& Tooke, W. (1988). The observational method: Studying the interaction of minds and bodies. In S. Duck (Ed.), The handbook of personal relationships (pp. 79-98). New York: Wiley.

Kenny, D. A., \& LaVoie, L. (1985). Separating individual and group effects. Journal of Personality and Social Psychology, 48, 339-348.

Kashy, D. (1991). Levels of analysis of social interaction diaries: Separating the effects of person, partner, day, and interaction. Unpublished doctoral dissertation, University of Connecticut.

McIntyre, C. W., Watson, D., \& Cunningham, A. C. (1990). The effects of social interaction exercise and test stress on positive and negative affect. Bulletin of the Psychonomic Society, 28, 141-143.

Nezlek, J. B., Imbrie, M., \& Shean, G. D. (1994). Depression and everyday social interaction. Journal of Personality and Social Psychology, 67, 1101-1111.

Pennebaker, J. W. ( 1989). Confession, inhibition, and disease. In M. P. Zanna (Ed.), Advances in experimental social psychology (Vol. 22, pp. 21 1-244). New York: Academic Press.

Reis, H. T., \& Wheeler, L. (1991). Studying social interaction with the Rochester Interaction Record. In M. P. Zanna (Ed.), Advances in ex- perimental social psychology (Vol. 24, pp. 270-318). New York: Academic Press.

Reis, H. T., Wheeler, L., Spiegel, N., Kernis, M., Nezlek, J., \& Perri, M. (1982). Physical attractiveness in social interaction, II: Why does appearance affect social experience? Journal of Personality and Social Psychology, 43, 979-996.

Schachter, S. (1959). The psychology of affiliation. Stanford, CA: Stanford University Press.

Tellegen, A. (1985). Structures of mood and personality and their relevance to assessing anxiety, with an emphasis on self-report. In A. H. Tuma \& J. D. Maser (Eds.), Anxiety and the anxiety disorders (pp. 681-706). Hillsdale, NJ: Erlbaum.

Watson, D. (1988a). Intraindividual and interindividual analyses of positive and negative affect: Their relations to health complaints, perceived stress, and daily activities. Joumal of Personality and Social Psychology, 54, 1020-1030.

Watson, D. (1988b). The vicissitudes of mood measurement: Effects of varying descriptors, time frames, and response formats on measures of positive and negative affect. Journal of Personality and Social Psychology. 55, 128-141.

Watson, D., \& Clark, L. A. ( 1984). Negative affectivity: The disposition to experience aversive emotional states. Psychological Bulletin, 96, 465-490.

Watson, D., \& Clark, L. A. (1992). On traits and temperament: General and specific factors of emotional experience and their relation to the five-factor model. Journal of Personality, 60, 441-476.

Watson, D., Clark, L. A., McIntyre, C. W., \& Hamaker, S. (1992). Affect, personality and social activity. Journal of Personality and SOcial Psychology, 63, 1011-1025.

Watson, D., Clark, L. A., \& Tellegen, A. (1988). Development and validation of brief measures of positive and negative affect: The PANAS scales. Journal of Personality and Social Psychology, 54, 1063-1070.

Watson, D., \& Pennebaker, J. W. (1989). Health complaints, stress, and distress: Exploring the central role of negative affectivity. Psychological Review, 96, 309-328.

Wheeler, L., \& Nezlek, J. ( 1977). Sex differences in social participation. Journal of Personality and Social Psychology, 35, 742-754.

Received August 9, 1995

Revision received January 24, 1996 Accepted January 30, 1996 